\title{
On the equivalence of finite difference and edge element formulations in magnetic field analysis using vector potential
}

\author{
Andrzej Demenko ${ }^{1}$, Jan K. Sykulski ${ }^{2}$, Fellow IEEE \\ ${ }^{1}$ Poznań University of Technology, POLAND, andrzej.demenko@put.poznan.pl \\ ${ }^{2}$ Electronics and Computer Science, University of Southampton, Southampton, UK, jks@soton.ac.uk
}

\begin{abstract}
Numerical three-dimensional formulations using vector potential $A$ are examined for magnetic fields, with emphasis on the finite-difference (FDM) and edge-element (EEM) methods. It is shown that for hexahedral elements the FDM equations may be presented in the form similar to the FEM equations, providing the products of the nodal potentials and distances between the nodes are used as unknowns in FDM, instead of the usual nodal potentials.
\end{abstract}

\section{INTRODUCTION}

Historically, the oldest numerical technique for modelling magnetic fields is the finite difference method (FDM) [1]. The contemporary view is that this method is unsuitable or impractical, especially for 3D fields when the vector potential $\boldsymbol{A}$ is used; it may therefore be worth noting that it is possible using the FDM formulation - to arrive at the final equations very similar to those obtained by the edge element method (EEM) for a mesh consisting of hexahedral elements.

The authors of this paper have had a long standing involvement with both FDM and EEM, the latter increasingly becoming a popular choice when vector potential is used in 3D simulation. Considering the pedagogical values of the FDM it is suggested therefore that the analogy between the two formulations should be explored and conditions for the equivalence of the final sets of equations investigated.

\section{The SUBSTITUTION $\boldsymbol{B}=C U R L \boldsymbol{A}$ IN FDM AND EEM}

Consider the substitution $\boldsymbol{B}=\operatorname{curl} \boldsymbol{A}$ and take as an example the $B_{x}$ and $B_{y}$ components. Using the FDM and with reference to Fig. 1 these components may be expressed as

$$
B_{x 1}=\frac{A_{z, y 2}-A_{z, y 1}}{\Delta y}-\frac{A_{y, z 2}-A_{y, z 1}}{\Delta z}, B_{y 1}=\frac{A_{x, z 2}-A_{x, z 1}}{\Delta z}-\frac{A_{z, x 2}-A_{z, x 1}}{\Delta x}
$$

Multiplying (1a) by $\Delta y \Delta z$ and (1b) by $\Delta x \Delta z$ yields $\phi_{x 1}=\varphi_{z, y 2}-\varphi_{z, y 1}-\varphi_{y, z 2}+\varphi_{y, z 1}, \phi_{y 1}=\varphi_{x, z 2}-\varphi_{x, z 1}-\varphi_{z, x 2}+\varphi_{z, x 1}$ where $\phi_{x 1}=B_{x 1} \Delta y \Delta z, \phi_{y 1}=B_{y 1} \Delta x \Delta z, \varphi_{u, v i}=A_{u, v i} \Delta u, i=1,2 ; u, v=x, y, z$. These FDM relationships may also be recognised as well known in EEM where they express the facet values of $\boldsymbol{B}$ (for the rectangular facets of Fig. 1) in terms of the edge values of $\boldsymbol{A}$, which in (2) are given by the products of the nodal values $A_{u, v i}$ and the distances between nodes $\Delta u$. It may therefore be argued that (2) represents the substitution $\boldsymbol{B}=\operatorname{curl} \boldsymbol{A}$ in EEM.


Fig.1. Graphical representation of the substitution $\boldsymbol{B}=$ curl $\boldsymbol{A}$ in FDM.

\section{EQUATION CURL $\boldsymbol{H}=\boldsymbol{J}$ IN FEM AND EEM}

Consider the finite difference form of $\operatorname{curlH}=\boldsymbol{J}$ for the $J_{z}$ component. For a uniform grid and with reference to Fig. 2a

$$
\left(H_{y, x 2}-H_{y, x 1}\right) / \Delta x-\left(H_{x, y 2}-H_{x, y 1}\right) / \Delta y=J_{z 0}
$$

Multiplying (3) by $\Delta x \Delta z$ results in

$$
u_{H y, x 2}-u_{H y, x 1}-u_{H x, y 2}+u_{H x, y 1}=\theta_{z 0}
$$

which may also be found in the EEM formulation, as the terms $u_{H v, w i}=H_{v, w i} \Delta v(i=1,2 ; v, w=x, y)$ are related to the edge values of vector $\boldsymbol{H}$, while $\theta_{z 0}$ represents the facet value of $\boldsymbol{J}$. From (3) and (4), after substituting $H_{v, w i}=v_{v i} B_{v i} \quad(i=1,2 ; v=x, y)$, and $B_{v i}=v_{v i} \phi_{v i} /(\Delta z \Delta v)$, where $v_{v i}=1 / \mu\left(P_{v i}\right)$, we find

$$
\phi_{y 2} R_{\mu y 2}-\phi_{y 1} R_{\mu y 1}+\phi_{x 1} R_{\mu x 1}-\phi_{x 2} R_{\mu x 2}=\theta_{z 0}
$$

This finite-difference derived relationship is equivalent to the loop equation of a magnetic circuit shown in Fig. 2b [2]. From (5) and substituting (2) for all nodes $P_{v i}$ we derive an equation analogous to the EEM formulation for the edge with its centre at $P_{0}$ (Figs 1 and 2). The apparent difference between (5) and the classical EEM scheme is due to the description of the terms $R_{\mu v i}$ representing the reluctances. In EEM these terms are derived from the integrals of the interpolating functions of the facet element. For the hexahedral elements, and employing the approximate integration described in [2], the resultant formulae will be similar to those of Fig. $2 b$.

\section{CONCLUSIONS}

It has been shown that by employing appropriate finitedifference forms the resultant FDM equations may be made identical to the EEM formulation for hexahedral elements; the relevant unknowns, $\varphi_{u, v i}$, are products of nodal potentials, $A_{u, v i}$, and distances between nodes, instead of the nodal potentials.

For non-homogenous and non-linear materials equivalent FDM and EEM equations may also be derived by defining reluctances $v_{v i}$ associated with points $P_{v i}$.

\section{REFERENCES}

[1]K.J. Binns, P.J. Lawrenson, C.W. Trowbridge, The analytical and numerical solution of electric and magnetic fields, J. Wiley, Chichester, 1992.

[2]A. Demenko, J.K. Sykulski, R. Wojciechowski, On the Equivalence of Finite Element and Finite Integration Formulations, IEEE Trans. on Magnetics, Vol. 46, No. 8, pp. 3169-72, 2010.
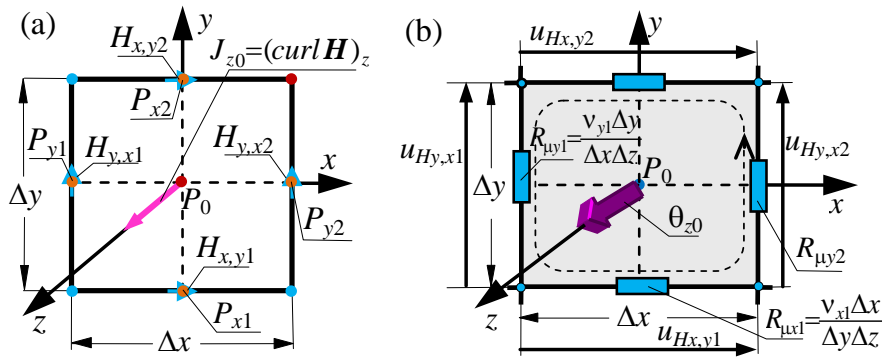

Fig.2. Finite difference (a) and circuit (b) representations of $\operatorname{curl} \mathbf{H}=\boldsymbol{J}$ 